\title{
An Investigation into the Role of Capping on Second Harmonic Generation from Nonlinear Organic Polymer and Guest-Host Thin Films by In-Situ Poling
}

\author{
P. M. Ushasree ${ }^{1^{*}}$, M. Szablewski ${ }^{2}$ \\ ${ }^{1}$ Department of Physics and Electrical Engineering, Northumbria University, Newcastle upon Tyne, UK \\ ${ }^{2}$ Department of Physics, Durham University, Durham, UK \\ Email: "palat.ushasree@northumbria.ac.uk
}

Received 26 March 2016; accepted 28 May 2016; published 31 May 2016

Copyright (C) 2016 by authors and Scientific Research Publishing Inc.

This work is licensed under the Creative Commons Attribution International License (CC BY).

http://creativecommons.org/licenses/by/4.0/

(c) (i) Open Access

\begin{abstract}
In attempts to fabricate thermally stable second-order nonlinear polymer thin films, we have investigated the second harmonic generation (SHG) from both nonlinear polymer and guest-host thin films. We have also investigated the role of capping on the SHG, temporal stability and relaxation of dipole alignment. Corona poling techniques were employed to orient the dopants into the noncentrosymmetric structure required to obtain the SHG. The effect of capping with a polymeric encapsulant below the glass transition temperature of the polymers on the unpoled and corona poled thin films was studied. Capping of the nonlinear polymer and guest host thin films have resulted in high SHG with good temporal stability. SHG signal falls drastically during the first 8 days after poling while no further significant decay in SHG signal was observed after about 33 days. Our investigations have identified the characteristics required for a good encapsulant on a non-conductive surface.
\end{abstract}

\section{Keywords}

Second Harmonic Generation, Nonlinear Polymer Thin Films, Guest-Host Thin Films, Capping, Temporal Stability, Corona Poling

\section{Introduction}

Polymers as optoelectronic materials have been widely studied [1]-[7]. In contrast to crystalline systems, these

${ }^{*}$ Corresponding author.

How to cite this paper: Ushasree, P.M. and Szablewski, M. (2016) An Investigation into the Role of Capping on Second Harmonic Generation from Nonlinear Organic Polymer and Guest-Host Thin Films by In-Situ Poling. Optics and Photonics Journal, 6, 101-111. http://dx.doi.org/10.4236/opj.2016.65013 
materials are cost effective and can be easily processed as thin films and utilised as device components for various applications such as frequency doubling for data storage and microlithography, bio-sensors and electro-optic modulation in telecommunications. The potential advantages of organic nonlinear polymers (NLP) include larger electro-optic coefficients, lower dielectric constants desirable for high speed devices, compatibility with different types of substrates and flexibility of fabrication methods [8]-[11]. Progress has been made in developing highly stable poled polymers [12]-[14]. However, the problem of temporal instability of the poled species remains. One approach is to conduct studies into relaxation mechanisms in polymers with a view to improve material stability.

Among the several techniques available for the fabrication of noncentrosymmetric nonlinear optical (NLO) thin films, the in-situ corona poling technique [15]-[17] has been used successfully to obtain the desired noncentrosymmetry, in this investigation. We studied the optical properties of spin coated thin films of three different kinds of organic NLO materials. One is an organic NLP; poly-dicyanovinyl Isophorone diisocyanate [pdcv-ipdi] and the other two are guest-host systems, DEMI (a zwitterionic TCNQ adduct) [18] and the complex salt of TCNQ and triethylamine; TEA (TCNQ) $)_{2}$ which can be converted into DEMI at around $160^{\circ} \mathrm{C}$ when in a polymer matrix, as shown in Figure 1(a) and Figure 1(b) [19]. DEMI and TEA (TCNQ) 2 were incorporated into a polymer host, polymethylacrylate (PMMA) for in-situ poling and SHG studies. To fabricate thermally stable organic NLP thin films, we have investigated the role of capping on SHG for [pdcv-ipdi], DEMI-PMMA and TEA (TCNQ) $)_{2}$-PMMA and a more detailed study of temporal stability and relaxation with time for [pdcv-ipdi] was carried out.

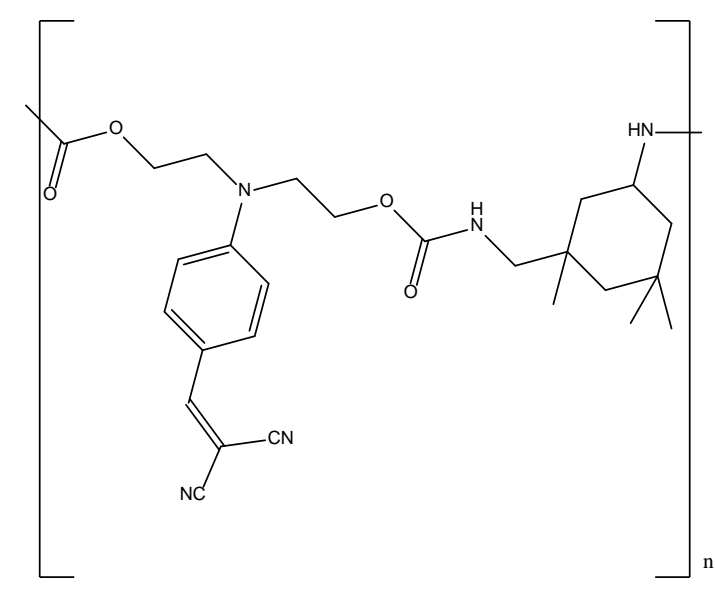

(a)
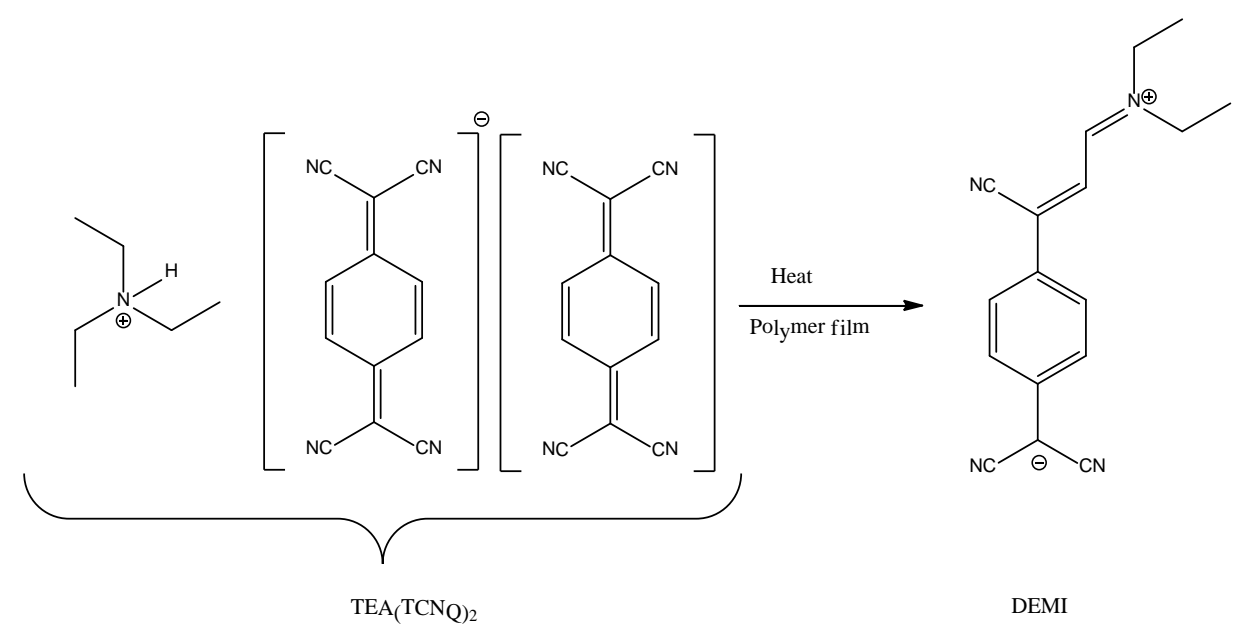

(b)

Figure 1. (a). Chemical structure of [pdcv-ipdi]; (b) conversion of TEA (TCNQ) ${ }_{2}$ into DEMI at around $160^{\circ} \mathrm{C}$ in a polymer matrix. 
There are reports which have observed physical damage on corona poled NLP films deposited on a conductive surface, such as an ITO-coated glass substrate [20] [21]. If the films are deposited on a non-conductive surface such as a glass substrate, some of the surface damage can be avoided [22]. The additional damage is unacceptable for electro-optic devices since it presents itself either as pinholes that cause electric shorts after the deposition of the second electrode or as surface roughness that causes scattering losses in waveguide devices [22]. In the present study, we have observed that films deposited on a non-conductive surface also experienced several problems and this has been easily avoided by depositing another polymer layer on the NLP or guest host system by means of spin-coating technique. This layer which we term as the capping layer or encapsulant, protects the nonlinear surface from any physical and chemical changes during in-situ poling and SHG. To our knowledge, there are only few reports on the role of capping on SHG, thermal stability and temporal stability or relaxation of SHG with time on a non-conductive surface [23].

This work aims to fabricate organic polymer thin films with large optical nonlinearities and to study their poling characteristics as well as both linear and NLO properties. Organic and guest-host thin polymer films have been deposited on glass substrates using the spin coating method. These polymer thin films are isotropic and thus are intrinsically centrosymmetric and incapable of exhibiting second order NLO effects.

In order to induce polar order to these guest-host systems different techniques can be employed. In the present investigation for [pdcv-ipdi], DEMI-PMMA and TEA (TCNQ) $)_{2}$-PMMA,we used the in-situ poling technique to measure the evolution of the normalised SHG signal while electrically corona poling the thin polymer film held at a temperature just above its glass transition temperature $\left(\mathrm{T}_{\mathrm{g}}\right)$ and on subsequent cooling. The corona poling technique provides a valuable insight into the build-up of polar orientation and stability of the alignment of NLO chromophores and is a very useful technique for screening NLPs with short and long orientation relaxation times. The in-situ technique developed here allows a characteristic profile to be recorded for each polymer or guesthost film, not only measuring the SHG as a function of poling field alignment and electric field, but also probing the dynamics of the poling and chromophore alignment behaviour.

\section{Experimental}

\subsection{Material Processing}

Poly-Dicyanovinyl Isophorone diisocyanate [pdcv-ipdi] was obtained from Chromophore, Inc. and the synthesis of DEMI and TEA (TCNQ) $)_{2}$ are described in our previous work [15] [16]. All the NLP thin films were deposited on a standard glass microscope slide by spin coating technique using a spin coater, single wafer spin processor, Model WS-400B-6NPP/LITE, Laurell Technologies Corporation. [pdcv-ipdi] is a yellow polymer with a glass transition temperature $\left(\mathrm{T}_{\mathrm{g}}\right)$ of $145^{\circ} \mathrm{C}$ and an absorption maximum at around $430 \mathrm{~nm}$. For thin film fabrication of [pdcv-ipdi], $0.5 \mathrm{~g}$ of the polymer [pdcv-ipdi] was dissolved in $4 \mathrm{ml}$ of cyclohexanone. The solution was filtered through a syringe with a $0.5 \mu \mathrm{m}$ filter attached and spin coated in steps of $500 \mathrm{rpm}$ for 2 seconds and $2000 \mathrm{rpm}$ for 10 seconds, onto the slide and dried immediately in a vacuum oven at $80^{\circ} \mathrm{C}$ for 17 hours to remove the residual solvent that might be trapped in the films.

The zwitterionic NLO chromophore, DEMI and the complex salt, TEA (TCNQ) $)_{2}$ were doped into PMMA, respectively. In the present investigation, thin films of DEMI-PMMA and TEA (TCNQ) 2 -PMMA were fabricated by codissolving DEMI and TEA $(\mathrm{TCNQ})_{2}$ with $1.5 \mathrm{~g}$ of PMMA $(\mathrm{MW}=100,00)$ in $5 \mathrm{ml}$ of anhydrous dimethylformamide (DMF) to yield films of $0.5 \mathrm{w} / \mathrm{w} \%$, respectively. The solutions were filtered with a $0.5 \mu \mathrm{m}$ filter and spin coated at the same spinning rate as above, onto a glass substrate and dried in a vacuum oven at $50^{\circ} \mathrm{C}$ for 17 hours to ensure complete removal of the solvent.

Subsequently, these films were then spin coated with an optically transparent capping layer. A choice of the material for the capping layer of its thickness is required in order to have reproducible conditions that favour poling. A capping layer acts as a medium into which the corona charges are deposited thereby creating the poling field. The charges are trapped in this layer and do not come directly into contact with the dopant molecules or polymers in the layer below where electrochemical damage could occur. The capping layer also helps prevent direct contact of the film with air and thus minimises photo-oxidation problems, as well as isolating it from adverse environmental conditions, such as carbon dioxide and moisture in the atmosphere. With respect to any free charges, this two layer system can be viewed as a pair of resistors in series. A good capping layer will thus be identified such that its $\mathrm{T}_{\mathrm{g}}$ will be less than that of the NLP thin films. At the poling temperature this should ensure that the resistance of the capping layer is much less than that of the doped layer which will then result in a 
high voltage across the NLP. Hence the requirements of a good capping layer are lower $\mathrm{T}_{\mathrm{g}}$ with respect to the film and good adhesion during poling. In the present investigation polyisobutene (PIB) and polyacyrilic acid (PAA) were chosen as capping materials and deposited on the NLP by spin coating. For this, $2 \mathrm{wt} \%$ of PIB $\left(\mathrm{T}_{\mathrm{g}}=\right.$ $75^{\circ} \mathrm{C}$ ) was dissolved in cycloctane and spin-coated on the NLP. A capping layer of PAA was fabricated by dissolving $12 \mathrm{wt} \%$ of PAA $\left(\mathrm{T}_{\mathrm{g}}=106^{\circ} \mathrm{C}\right)$ in de-ionized water. As the decomposition temperature of the [pdcv-ipdi] is higher than the corresponding $\mathrm{T}_{\mathrm{g}}$, high temperature poling for a short period of time is possible without damaging the NLO chromophore.

\subsection{Measurements}

To induce noncentrosymmetric polar order in the spin coated polymer films, the films were corona poled using an in-situ poling technique, a schematic diagram of which is shown in Figure 2. Corona poling is the most versatile technique for poling polymer films [15]-[17]. The technique involves applying a high voltage, typically about 5 - $10 \mathrm{KV}$ between a needle and a conducting bottom electrode. The poling field is then created due to the ionized charges, generated from a corona discharge, deposited on the surface of the polymer thin film. The laser source used for generating SHG is a Q-switched Nd: YAG pulse laser with a $1064 \mathrm{~nm}$ fundamental beam and 30 $\mathrm{Hz}$ repeating rate. Normalised SHG was measured as a function of applied temperature while simultaneously corona poling the thin films. The thin films were heated to few degrees above their respective $\mathrm{T}_{\mathrm{g}}$ at a rate of $200^{\circ} \mathrm{C} / \mathrm{hr}$. and held at that temperature for about 15 minutes. Until their respective $\mathrm{T}_{\mathrm{g}}$ were attained i.e., during the increase in temperature, the poling field was alternatively applied and removed in order to study the effect of poling at temperatures below $\mathrm{T}_{\mathrm{g}}$ for capped and uncapped thin films. The poling field was then applied continuously when approaching the $T_{g}$ and until the film was cooled back to room temperature. Close to the $T_{g}$, the molecular mobility of the molecules increases and allows the alignment of the dipoles during poling. When the thin films are cooled to room temperature, the molecules are frozen into their new orientation.

To evaluate the high-temperature stability of the PAA capped organic polymer system, [pdcv-ipdi], the temporal stability of dipole orientation of the poled [pdcv-ipdi], after the removal of the poling field, as a function of temperature was studied. To monitor the decay of SHG with temperature, the poled [pdcv-ipdi] film was heated at a heating rate of $3.3^{\circ} \mathrm{C} / \mathrm{min}$ from $21^{\circ} \mathrm{C}$ to $150^{\circ} \mathrm{C}$ and the decay in SHG intensity was recorded.

To reveal the poling characteristics, absorption spectra of the unpoled and poled capped thin films were measured using a PERKIN-Elmer Lambda 19UV/Vis spectrometer. To study the relaxation of dipole alignment of PAA capped thin film of [pdcv-ipdi] for long term thermal stability, the SHG was measured just after poling and for a successive number of days at room temperature. The effect of a capping layer was further studied for the other two guest-host systems, namely DEMI and TEA (TCNQ) 2 by a similar method.

\section{Results and Discussion}

The NLO properties of the polymer [pdcv-ipdi], with and without capping were studied by in-situ corona poling. All the thin films were mounted on the sample holder for simultaneous corona poling and SHG measurements (Figure 2). Figures 3(a)-(c) show the evolution of the normalised SHG as a function of temperature for uncapped,

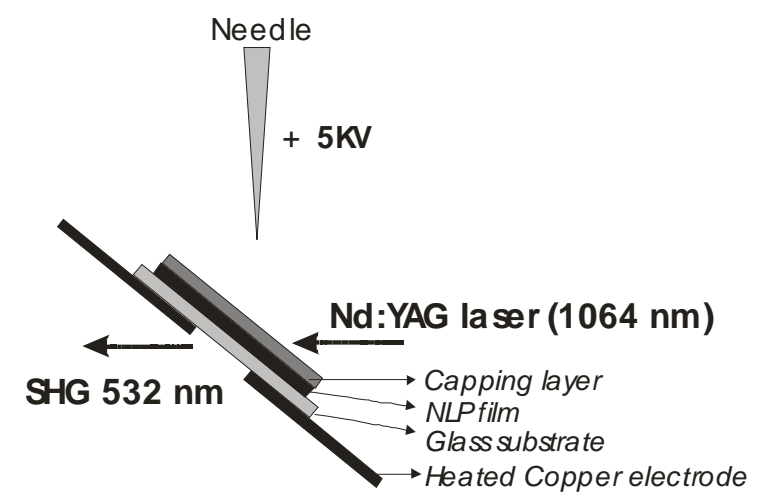

Figure 2. Schematic diagram of the in-situ poling technique. 

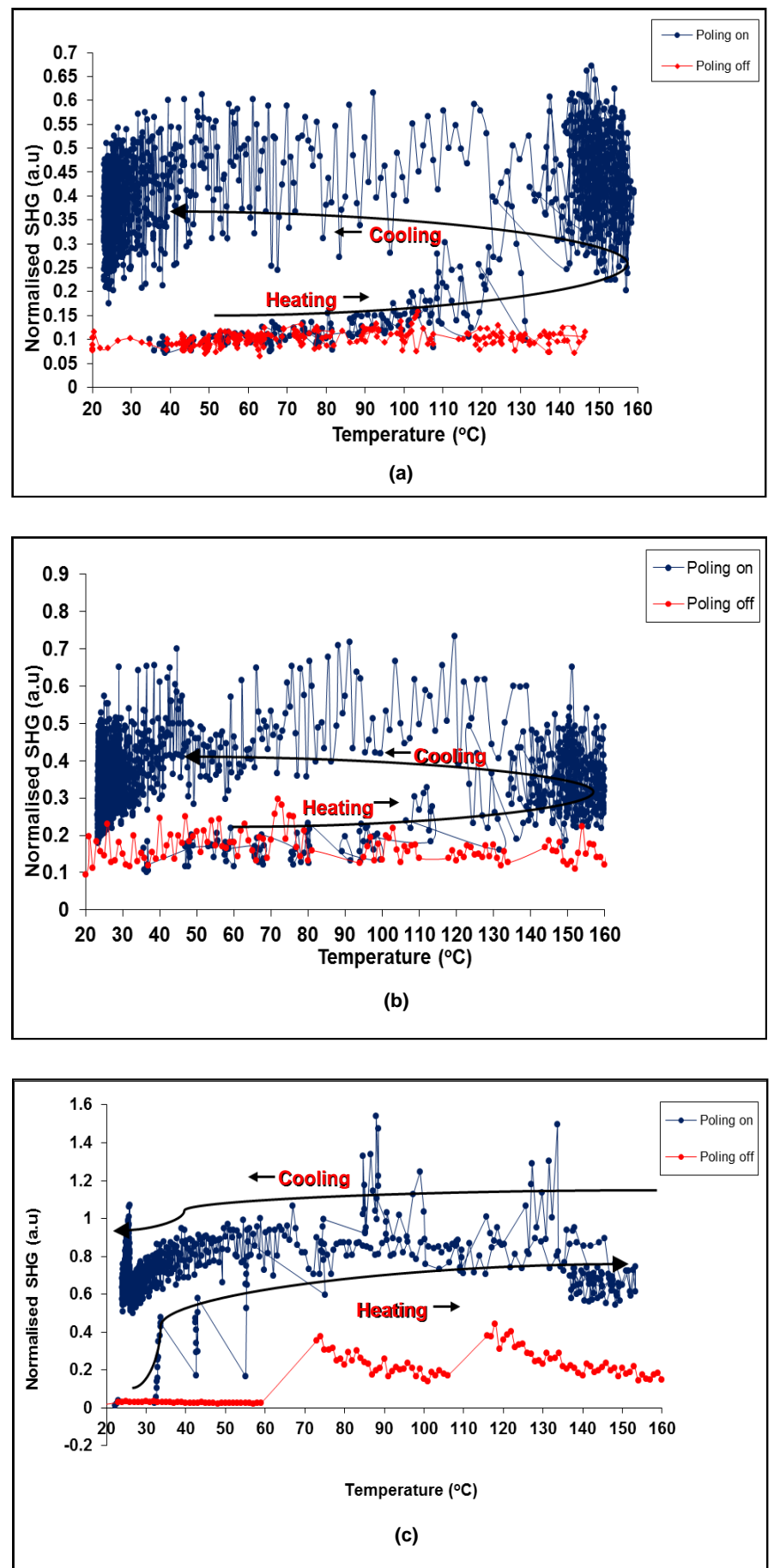

Figure 3. Poling characteristics of (a) uncapped, (b) PIB capped and (c) PAA capped [pdcv-ipdi] thin film (line joining the points are a guide to the eye).

PIB and PAA capped [pdcv-ipdi] thin film, respectively. The red dots represent the normalised SHG as a function of temperature when the poling field is switched off. The blue dots represent the normalised SHG as a function of temperature when the poling field is switched on. For the PIB capped thin film [pdcv-ipdi], the normalised SHG intensity increases to a slight amount. For the PAA capped thin film of [pdcv-ipdi], the normalised SHG increased considerably even at lower temperatures. It was observed that at lower temperatures, for the 
PAA capped [pdcv-ipdi], as the poling field is applied the normalised SHG increases and then falls as the poling field is removed. Finally, when nearing the $T_{\mathrm{g}}$, when the poling field was applied continuously until the film was cooled back to room temperature, the normalised SHG remained high and did not reduce because of the fixed 'locked in' alignment of dipole moments in their new orientation. This may be due to surface charges being trapped in the capping layer causing a tremendous increase in normalised SHG intensity even at lower temperatures below the $T_{\mathrm{g}}[14]$.

Similar measurements were carried out with DEMI-PMMA and TEA (TCNQ) 2 -PMMA thin films. Figure 4(a) and Figure 5(a) show normalised SHG as a function of temperature of uncapped DEMI-PMMA and TEA $(\mathrm{TCNQ})_{2}$-PMMA. It was observed that for uncapped DEMI-PMMA and TEA (TCNQ) $)_{2}$-PMMA, the normalised SHG falls down during cooling. PIB capped DEMI-PMMA and TEA (TCNQ) $)_{2}$-PMMA showed a similar behaviour. This may be due to evaporation of DEMI-PMMA and TEA (TCNQ) 2 -PMMA at high temperatures indicating that PIB is not an efficient capping layer for these systems. It was observed that for the capped DEMI-PMMA and TEA (TCNQ)2-PMMA thin films, as shown in Figure4(b) and Figure 5(b), even at lower temperatures there was an increase in normalised SHG, which clearly indicates that PAA is in these experiments an efficient capping layer.

Figure 6(a) shows the absorption spectra of unpoled and poled, DEMI. After poling, a decrease in absorbance was observed due to alignment of the chromophore and surface charge accumulation. Figure 6(b) shows the absorption spectra of unpoled and poled TEA (TCNQ) $)_{2}$. In the absorption spectra of poled TEA (TCNQ) $)_{2}$, the
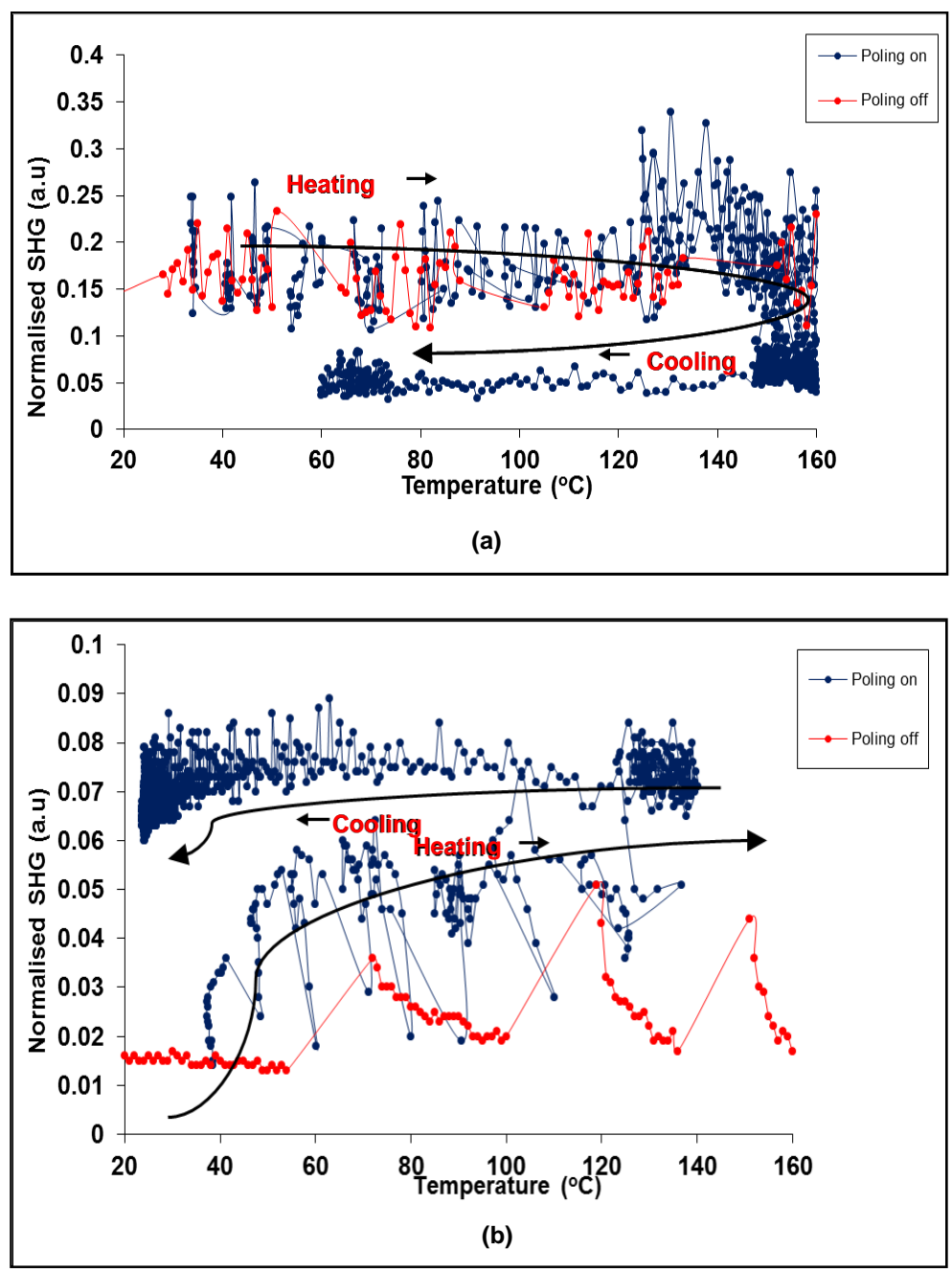

Figure 4. Poling characteristics of (a) uncapped and (b) PAA capped DEMIPMMA thin film (line joining the points are a guide to the eye). 

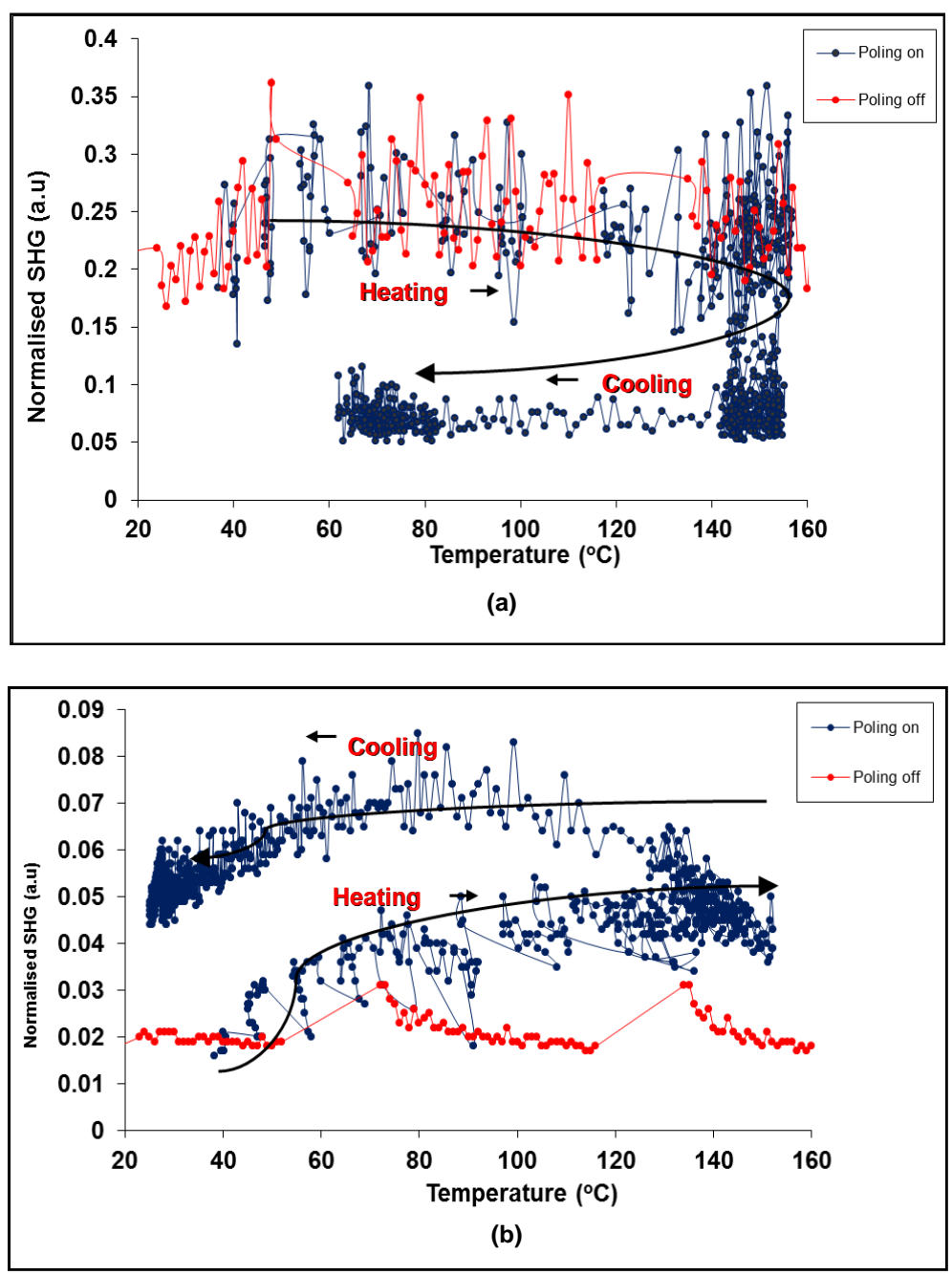

Figure 5. Poling characteristics of (a) uncapped and (b) PAA capped TEA (TCNQ)2-PMMA thin film (line joining the points are a guide to the eye).

intensity of the peak due to neutral TCNQ at lower wavelength, $403 \mathrm{~nm}$ decreases and TCNQ — at higher wavelengths, 685 and $749 \mathrm{~nm}$ disappears while the peak intensity at $850 \mathrm{~nm}$ decreases. New peaks due to DEMI in PMMA at wavelengths, 656 and $749 \mathrm{~nm}$ arises. This clearly shows the conversion of TEA (TCNQ) ${ }_{2}$-PMMA film into DEMI-PMMA film at around $160^{\circ} \mathrm{C}$. This is in line with our previous findings [19].

Thermal stability of the dipole orientation has been studied and relaxation of dipole alignment with time for [pdcv-ipdi] is investigated. Figure 7 shows the decay of SHG intensity as a function of temperature for the thin film of [pdcv-ipdi]. It was observed that the polymer thin film was quiet stable upto $120^{\circ} \mathrm{C}$, around $25^{\circ} \mathrm{C}$ below its $\mathrm{T}_{\mathrm{g}}$. When the thin film of poled [pdcv-ipdi] was heated above $120^{\circ} \mathrm{C}$, the SHG signal dropped rapidly and disappeared at $\mathrm{T}_{\mathrm{g}}$. This is because unlike main chain polymers, the side chain polymers have lower thermal stability of dipole alignments at higher temperatures.

The decay of SHG signal as a function of time has been measured. Figure 8 shows the long term thermal stability of SHG for [pdcv-ipdi], when the films were stored at room temperature in the dark for the time of about 60 days. It has been observed that SHG signal falls drastically during the first 8 days after poling while no further significant decay in SHG signal was observed after about 33 days. This shows that the polymer has good long term thermal stability of SHG with no significant relaxation at ambient condition in 33 days. To investigate the decay of SHG signal with time, the absorption spectra of the unpoled, just after poling and 33 days after poling were taken and are shown in Figure 9. For the unpoled film the absorption peak, $\lambda_{\max }$ was observed at $429 \mathrm{~nm}$. Just after poling the intensity of $\lambda_{\max }$ decreased, but after 33 days the intensity of $\lambda_{\max }$ increased. This 

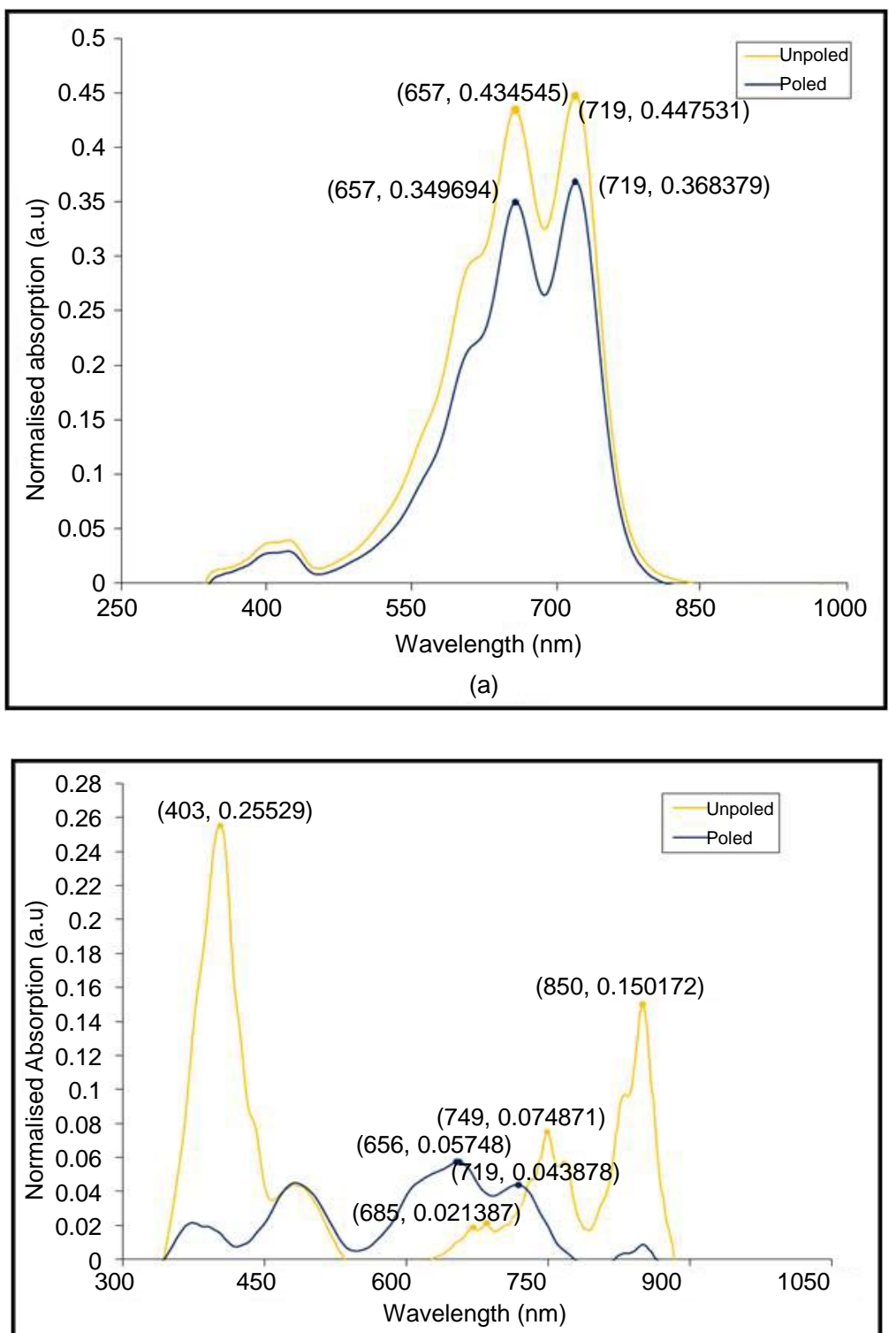

(b)

Figure 6. Absorption spectra of (a) DEMI and (b) TEA (TCNQ)2 before and after poling, respectively.

implies that during 33 days after poling both induced dipole interaction due to the surface charges and the dipole orientation were present but 33 days after poling the surface charge had dissipated and the SHG due to dipole orientation remains stable there after. The shape of the absorption spectrum before and after poling remained same which indicates that the decrease in absorption is not due to any chemical change during poling.

The SHG intensity for a thin film of [pdcv-ipdi] was measured as a function of incidence angle. SHG intensity of the thin film changes with the incident angle as shown in Figure 10. The minimum value was observed at $0^{\circ}$, while the maximum was observed at around $52^{\circ}$ because of the isotropic distribution of dipole moments and their projections within the thin film surface [22].

\section{Conclusion}

A noncentrosymmetric polar order was induced into fabricated NLP thin films by in-situ corona poling while simultaneously measuring the SHG using Nd: YAG laser of wavelength $1064 \mu \mathrm{m}$. The effects of capping with 


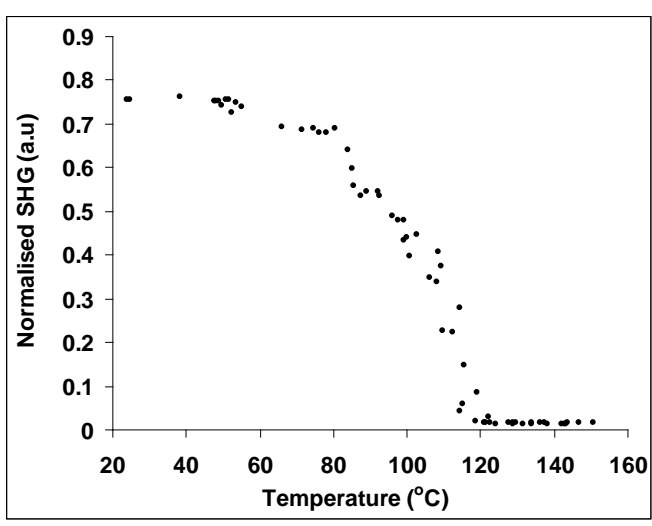

Figure 7. Decay of SHG of PAA capped [pdcv-ipdi] as a function of temperature at a heating rate of $3.3^{\circ} \mathrm{C} / \mathrm{min}$ (error bars are too small to be seen).

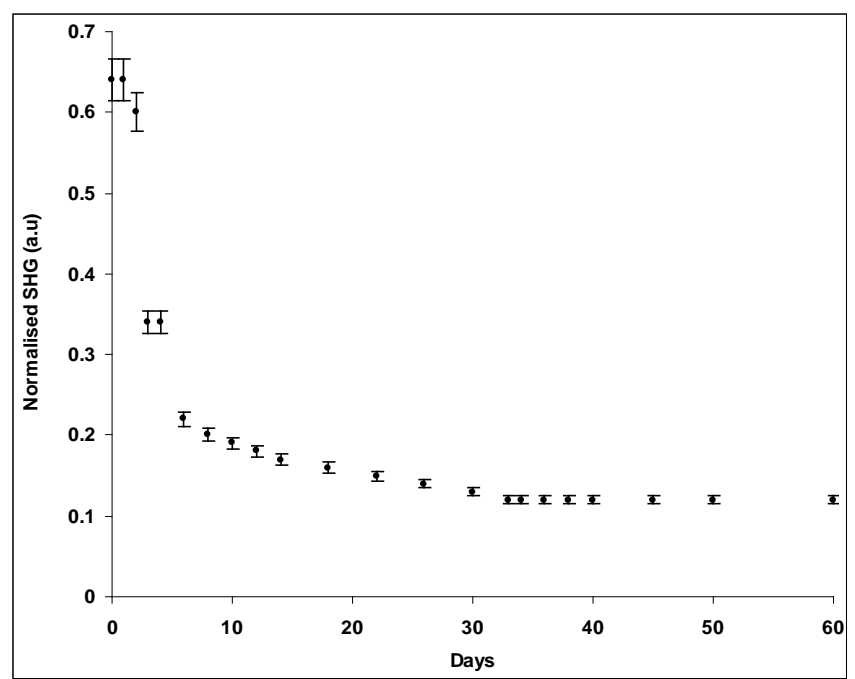

Figure 8. Long term thermal stability of SHG of [pdcv-ipdi] thin film stored at room temperature.

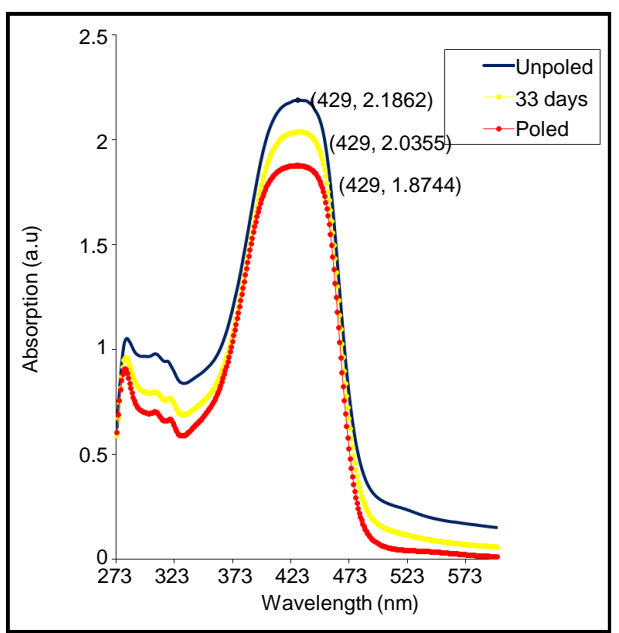

Figure 9. Absorbance spectra of capped [pdcv-ipdi] before and after poling. 


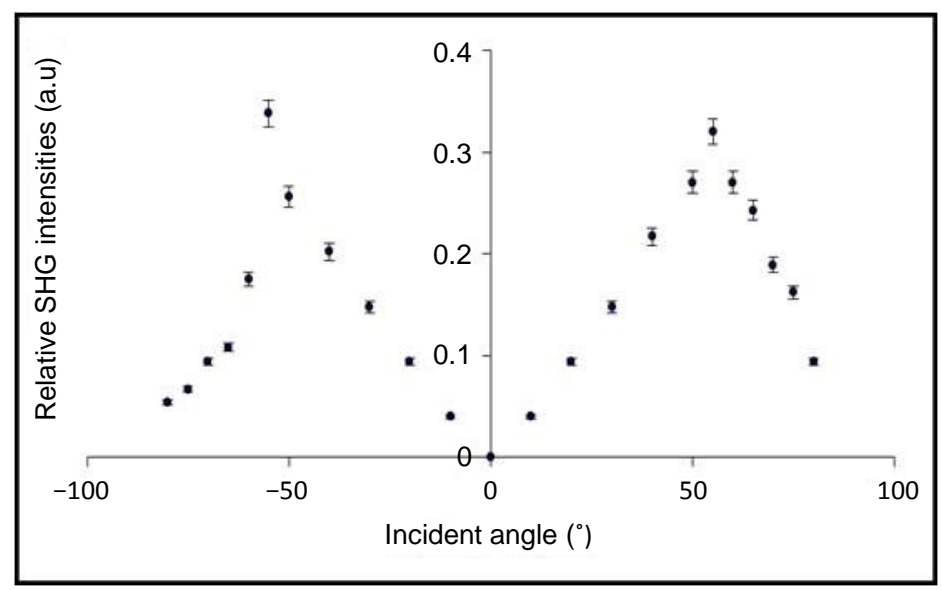

Figure 10. Change in SHG intensity with incident angle in [pdcv-ipdi] thin films.

PIB and PAA on SHG for polymer thin films were studied. Good thermal stability of nonlinearity was observed for the capped and poled thin films of [pdcv-ipdi] at room temperature in contrast with similar uncapped films. In the course of the experiments, the thermal stability of [pdcv-ipdi] was monitored using UV/VIS absorption spectra. For the PAA capped thin films, even at lower temperatures, the normalised SHG remained high and did not reduce because of the fixed "locked in" alignment of dipole moments in their new orientation. Capping of the NLP thin films with PAA resulted in high SHG with good temporal stability, showing that this system is more efficient than PIB as a capping material. Similar effects were observed for capped and uncapped organic NLO guest-host systems. The results obtained from studies of NLO stability investigations demonstrate the possibility of designing efficient PAA capped NLP thin films for large variety of systems, devices and applications.

\section{References}

[1] Prasad, P.N. and Williams, D.J. (1991) Introduction to Nonlinear Optical Effects in Molecules and Polymers. Wiley, New York.

[2] Marder, S.R., Kippelen, B., Jen, A.K.Y. and Peyghambarian, N. (1997) Design and Synthesis of Chromophores and Polymers for Electro-Optic and Photorefractive Applications. Nature, 388, 845.

[3] Shi, Y., Zhang, C., Bechtel, J.H., Dalton, L.R., Robinson, B.H. and Steier, W.H. (2000) Low (Sub-1 Volt) Halfwave Voltage Electrooptic Polymer Modulators Achieved by Controlling Chromophore Shape. Science, 288, 119.

[4] Lee, M., Katz, H.E., Erben, C., Gill, D.M., Gopalan, P., Heber, J.D. and McGee, D.J. (2002) Broadband Modulation of Light by Using an Electro-Optic Polymer. Science, 298, 1401.

[5] Garner, S.M., Cites, J.S., He, M.Q. and Wang, J.G. (2004) Polysulfone as an Electro-Optic Polymer Host Material Applied Physics Letters, 84, 1049. http://dx.doi.org/10.1063/1.1646754

[6] Kajzar, F., Lee, K.S. and Jen, A.K.Y. (2003) Polymeric Materials and Their Orientation Techniques for Second-Order Nonlinear Optics. Advances in Polymer Science, 161, 1. http://dx.doi.org/10.1007/3-540-45642-2_1

[7] Enami, Y., Meredith, G., Peyghambarian, N., Kawazu, M. and Jen, A.K.Y. (2003) Hybrid Electro-Optic Polymer and Selectively Buried Sol-Gel Waveguides. Applied Physics Letters, 82, 490. http://dx.doi.org/10.1063/1.1539298

[8] Wang, N.P., Leslie, T.M., Wang, S. and Kowel, S.T. (1995) Syntheses of Second-Order Nonlinear Optical Polyurethanes for Electrooptic Etalons. Chemistry of Materials, 7, 185-191. http://dx.doi.org/10.1021/cm00049a028

[9] Tumolillo, T.A. and Ashley, P.R. (1992) A Novel Pulse-Poling Technique for EO Polymer Waveguide Devices Using Device Electrode Poling. IEEE Photonics Technology Letters, 4, 142-145. http://dx.doi.org/10.1109/68.122342

[10] Ashley, P.R. and Tumolillo, T.A. (1991) Overview of EO Polymers for Guided-Wave Devices. Proceedings of the SPIE, Integrated Optical Circuits, 1583, 316. http://dx.doi.org/10.1117/12.50901

[11] Lytel, R., Lipscomb, G.F., Kenney, J.T. and Ticknor, A.J. (1991) Applications of Electro-Optic Polymers to Optical Interconnects. Proceedings of the SPIE, Optical Enhancements to Computing Technology, 1563, 122. http://dx.doi.org/10.1117/12.49675

[12] Jen, A.K.Y., Wong, K.Y., Pushkara Rao, V., Drost, K. and Cai, Y.M. (1994) Thermally Stable Poled Polymers: Highly 
Efficient Heteroaromatic Chromophores in High Temperature Polymides. Journal of Electronic Materials, $23,653$.

[13] Cui, Y., Qian, G., Chen, L., Wang, Z., Gao, J. and Wang, M. (2006) Enhanced Thermal Stability of Dipole Alignment in Inorganic-Organic Hybrid Films Containing Benzothiazole Chromophore. The Journal of Physical Chemistry B, 110, 4105-4110. http://dx.doi.org/10.1021/jp057146a

[14] Samoc, A., Holland, A., Tsuchimori, M., Watanabe, O., Samoc, M., Luther-Davies, B. and Kolev, V.Z. (2005) In Situ SHG Monitoring of Dipolar Orientation and Relaxation in Disperse Red Type/Derivative Urethane-Urea Copolymer. Proceedings of the SPIE, Nonlinear Optics Applications, 5949, 191. http://dx.doi.org/10.1117/12.622712

[15] Hill, R.A., Knoesen, A. and Mortazavi, M.A. (1994) Corona Poling of Nonlinear Polymer Thin Films for Electro-Optic Modulators. Applied Physics Letters, 65, 1733. http://dx.doi.org/10.1063/1.112899

[16] Hampsch, H.L., Torkelson, J.M., Bethke, S.J. and Grubb, S.G. (1990) Second Harmonic Generation in Corona Poled, Doped Polymer Films as a Function of Corona Processing. Journal of Applied Physics, 67, 1037. http://dx.doi.org/10.1063/1.345787

[17] Mortazavi, M.A., Knoesen, A., Kowel, S.T., Higgins, B.G. and Dienes, A. (1989) Second-Harmonic Generation and Absorption Studies of Polymer-Dye Films Oriented by Corona-Onset Poling at Elevated Temperatures. Journal of the Optical Society of America B, 6, 733-741. http://dx.doi.org/10.1364/JOSAB.6.000733

[18] Szablewski, M. (1994) Novel Reactions of TCNQ: Formation of Zwitterions for Nonlinear Optics by Reaction with Enamines. Journal of Organic Chemistry, 59, 954-956. http://dx.doi.org/10.1021/jo00084a008

[19] Szablewski, M. and Cross, G.H. (2005) In-Situ Synthesis and Alignment by Poling of a Zwitterionic NLO Chromophore in a Polymeric Guest-Host System. Proceedings of the SPIE, Nonlinear Optics Applications, 5949, 595902-1. http://dx.doi.org/10.1117/12.622746

[20] Herminghaus, S., Smith, B.A. and Swalen, J.D. (1991) Electro-Optic Coefficients in Electric-Field-Poled Polymer Waveguides. Journal of the Optical Society of America B, 8, 2311-2317. http://dx.doi.org/10.1364/JOSAB.8.002311

[21] Morichere, D., Chollet, P.A., Fleming, W., Jurich, M., Smith, B.A. and Swalen, J.D. (1993) Electro-Optic Effects in Two Tolane Side-Chain Nonlinear-Optical Polymers: Comparison between Measured Coefficients and SecondHarmonic Generation. Journal of the Optical Society of America B, 10, 1894-1900. http://dx.doi.org/10.1364/JOSAB.10.001894

[22] Rau, I. and Kajzar, F. (2008) Second Harmonic Generation Technique and Its Applications. Nonlinear Optics and Quantum Optics, 38, 99.

[23] Shi, W., Ding, Y.J., Fang, C., Pan, Q. and Gu, Q. (2003) Investigation of Charge Effects on Poling and Stability for Corona-Poled Polymer Films. Applied Physics A, Materials Science and Processing, 77, 567-570. http://dx.doi.org/10.1007/s00339-002-1499-0 